\title{
THE CHEMICAL INHIBITION OF FIBRE REGENERATION AND NEUROMA FORMATION IN PERIPHERAL NERVES
}

\author{
BY
}

\author{
L. GUTTMANN and P. B. MEDAWAR
}

From the Department of Zoology and Comparative Anatomy, University of Oxford

(Received 25Th SePtember, 1942)

\section{Introduction}

THE problem of suppressing the regenerative growth of nerve fibres by chemical or other means owes its clinical interest to the need for preventing or stopping the acute pain that sometimes develops after injury to peripheral nerves. The conditions under which acute pain develops have not been closely defined. It cannot be definitely attributed to any one particular cause, and it is not invariably accompanied be neuroma formation. Reactions from islands of infection and foreign bodies in the nerve itself (Marinesco 1918, 1920), the involvement of escaping nerve fibres in scar tissue and muscle (discussed by Corner, 1918b), and pressure upon "young" unmyelinated fibres (Cone, 1918) may each play a part. Clinical evidence nevertheless shows that pain in some form, particularly in amputation stumps, is associated with neuroma formation and other consequences of unorganized regenerative growth. Pain of this sort is sometimes relieved by excising the neuroma, and is prevented from recurring, or from arising in the first instance, by taking certain preventative measures. These may be reviewed shortly under two headings:

\section{A. Surgical and Mechanical}

Diversion of the free end of the nerve, with or without resection, into a position where it is protected by muscle bellies and free from violent pressure and movement ; or into bone (Seddon, 1941), where it may come to no harm. Implantation of the central stump into muscle (Moscowicz, 1918). Crushing (Kruger, 1916) or ligating the central stump. Closure of the open end of the central stump by ligating an epineurial sleeve slipped over the end of the nerve (Chapple, 1918), or by suturing the "trapdoor" flaps left after excising a wedge-shaped piece from the tip (Corner, 1918a). Reflection of the nerve into or through itself (Bardenhäuer, 1908*).

The more radical of these methods have been severely criticized by Huber and Lewis (1920), Stookey (1922), and others. At the other extreme, leaving the nerve as far as possible alone may be regarded as a calculated surgical procedure.

* We have tried to examine the rationale of Bardenhäuer's and similar operations by a series of experiments in which the central stumps of the tibial and peroneal divisions of the sciatic nerve of the rabbit were sutured into each other through an intermediate stretch of autograft. Multiple bulb formation with gross fibre abnormalities accompanied by whorling and deviation of the fibre bundles were the rule. We found no evidence that regenerating fibres could enter a common endoneurial pathway from opposite ends and block each other in the middle, although from what is known of fibre regeneration rates in rabbits (Young and Medawar, 1940; Sanders and Young, 1942; Gutmann, Guttmann, Medawar, and Young, 1942) they should in theory have had an opportunity to do so.

\section{B. Physical and Chemical}

Coagulation by cauterization or the intraneural injection of hot water has been described by Hedri (1921), Erlacher (1924), and Lexer (1931). Laewen (1925, 1930) claims that freezing the sciatic nerve in man for 4 to 6 minutes abolishes conduction for $1 \frac{1}{2}$ to 2 years, though interruption by cold is more generally regarded as being only temporary in effect.

The chemical reagents injected into nerves for the relief of pain and for preventing neuroma formation belong, with few exceptions, to the class of coagulants. Ethyl alcohol has been used for the treatment of causalgias (Sicard, 1916), neuralgias (Schlosser, 1903; Harris, 1909 ; Patrick, 1912 ; May, 1912), and for the injection of amputation stumps (Sicard, 1916; Huber and Lewis, 1920 ; Huber, 1927 ; Foerster, 1929 ; Beswerschenko, 1929). Experimental and histological studies of its action on nerve have been made by Finkelnburg $(1903,1907)$, May (1912), Bersou (1912); the most detailed and complete analysis is that of Huber and Lewis (1920) reported with full protocols by Huber (1927). Other fixatives and coagulants whose effects on nerve regeneraㅡㅡㅁ tion have been studied experimentally or clinically ${ }^{\mu}$ include: Osmium tetroxide (osmic acid), Neuber, 1883; Fraenkel, 1884. Mercuric chloride (mercury perchloride), Erlacher, 1924. Phenol (carbolic acid), Benedict, 1922 ; Frankenthal, 1928 ; Beswerschenko, 1929. Formaldehyde, Erlacher, 1924 ; Foerster, 1929 ; Beswerschenko, 1929.

The procedures reviewed under headings $\mathrm{A}$ and $\mathrm{B}$ have different objects. Resection of the stump or insertion into bone may be thought of as methods for putting the nerve out of harm's way. Ligation, the surgical closure of the end of the stump, and Bardenhäuer's operation are designed to prevent the escape of fibres, since they will certainly not prevent neuroma formation. The chemical methods are the most radical, since their object is to abolish living fibres from the terminal stretch of the nerve.

The experiments described in this paper, which follow in main outline those of Huber and Lewis (Huber, 1927), are chiefly concerned with the injection of chemical substances into the tibial division of the sciatic nerve of the rabbit. Their main object is to find out which reagents among a range of reasonable choice inhibit the regenerative growth of axons for the longest time. There is no ground for believing that permanent inhibition can be brought about by any method not involving the destruction of the cell-body of the neurone. Isolated clinical examples (e.g. Dustin, 1918) and animal experiments (Holmes and Young, 1942) show that the central stump of a divided nerve retains its power to reinnervate the periphery for a long time after 
division; and while this power may very well decline over the whole life span, as the power of repair in general is known to do, the decline can hardly be significant from a clinical point of view. A second object of these experiments has been to examine the relationship between axon regeneration, neuroma formation, and the tendency of nerve to become heavily involved in and adherent to the tissues in its neighbourhood; and in laboratory animals, it is possible to make a clearer distinction between the effects of nerve damage and those of collateral injury than is possible by clinical observation. On the other hand, the problem of the origin of pain cannot be solved by animal experiments alone.

\section{The Etiology of Neuroma Formation}

A well-marked swelling almost invariably forms at the end of the central stump of a divided nerve, though it may later subside. Under favourable circumstances, the development of this swelling may be only incidental to the process of repair after an operation of grafting or suture, but the regenerative growth of fibres takes place irrespective of whether they regain their normal path of growth or not. The formation of a central end bulb is the response of growing fibres to interference in their path of growth. Careful pinching of a nerve may interrupt all the fibres while creating no serious obstacle to their subsequent regeneration. Under these circumstances, no central swelling is formed. On the other hand, the constriction of a nerve with a very tight ligature (Fig. 1, A) invariably leads to the formation of a local swelling immediately central to the point of ligation. If two such ligatures are tied 10 to $20 \mathrm{~mm}$. apart in such a way that no fibres can escape through the upper one, or if the nerve is interrupted without section by applying a drop of chromic acid well above a single ligature, then no swelling develops at the lower interruption (Fig. 1, b). The experiments on chemical inhibition described below confirm and reinforce the fact that in the absence of fibres, a central end bulb is not formed, although there is no reason why we should not expect a swelling to form

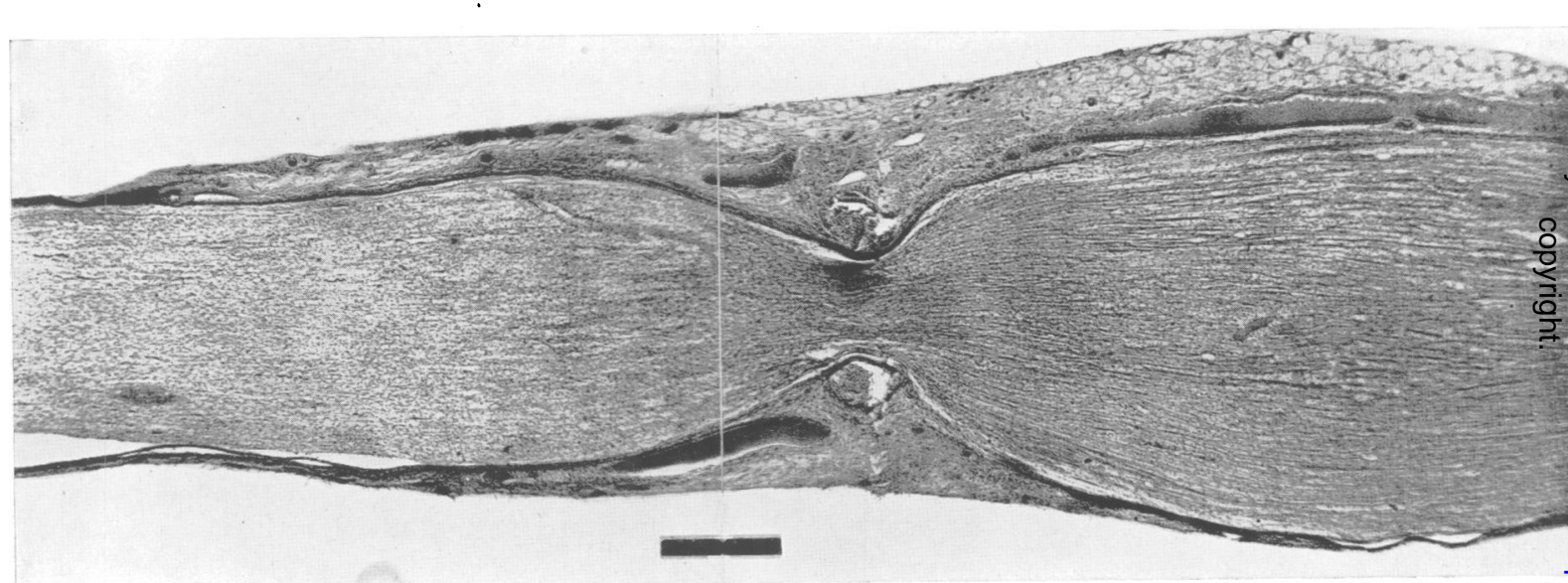

A

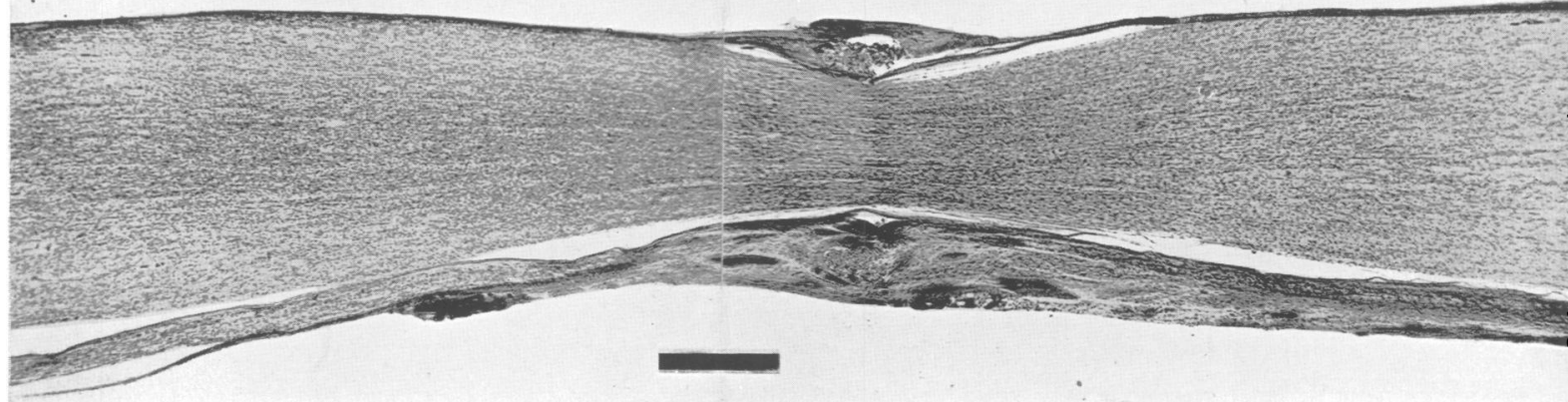

B

FIG. 1.-(A) 25 days after tying a tight ligature round the tibial nerve of the rabbit: central and peripheral swellings about the plane of the ligature. Regenerating fibres have just begun to force their way through the hour-glass constriction.

(B) 25 days after tying a similar ligature round a stretch of nerve which has been " sterilized" by tying a tight ligature central to the one illustrated here. No swellings form.

The central stump lies to the right. Scale $0.5 \mathrm{~mm}$. Bodian's stain. 
by the proliferation of endoneurial and other connective tissue elements, and in particular of Schwann cells. The problems of inhibiting neuroma formation and of suppressing the regenerative growth of axons, which Stookey distinguishes, are therefore coextensive in scope.

\section{Experiments on Chemical Inhibition}

Technique: The sciatic nerve of the rabbit is exposed by an incision parallel to and just inside the margin of the femur, and the tibial division separated from the peroneal down the length so exposed. The tibial is raised and packed with moist gauze around all surfaces except the upper. In this position the nerve can be safely injected with 0.1 to $0.2 \mathrm{ml}$. of fluid, and either subepineurally or intraneurally. In the former case, the injection fluid travels $60 \mathrm{~mm}$. or more along the highly permeable epineurial lymph spaces and destroys a corresponding length of nerve. In the latter, the injection fluid forms a localized bulb, which does not usually collapse when the needle is withdrawn, and which forms a local reservoir of high concentration. After a single injection by one or the other of these methods, the nerve is cut at, or not more than $10 \mathrm{~mm}$. below the injection point, and replaced parallel to but not in contact with the peroneal. In a few cases (cf. Huber, 1927) the nerve has been left uncut after injection in order to give the central stump the most favourable chance, under the circumstances, of reinnervating the periphery.

In the majority of experiments, the animals were reexamined after two months, a period more or less arbitrarily chosen in the first place as the earliest at which an indicative result could be expected. If a neuroma had formed after two months, it was excised and examined histologically. If not, the animals were allowed to survive for periods up to and in many cases exceeding a year.

Controls: The behaviour of the sciatic nerve of the rabbit after simple section and resection has been studied by Huber and Lewis (1920) and reported in detail by Huber (1927). An extensive series of neuromas formed within 50 days of section are available from other experiments done in this department; and in addition to these, twelve long-period controls have been laid down. They differ from those of Huber and Lewis in being concerned only with the tibial division of the sciatic nerve, in extending over a wider range of time (103 to 457 days), and in the relatively large gaps (30 to $40 \mathrm{~mm}$.) left between the stumps after resection. These controls, taken in conjunction with the experiments using reagents which proved to be ineffective, make it possible to draw a clear-cut distinction between " successful " and unsuccessful inhibitions (see below, p. 134).

Histological techniques : After fixation by the formolalcohol-acetic mixture recommended by Bodian (1936) or by formol saline, serial longitudinal sections of the histological material have been stained by the methods of Bodian or Holmes (1942) respectively for the demonstration of axons. Where sufficient material has been available, alternate slides have been stained or counterstained by hæmatoxylin and eosin or Masson's trichrome stain.

The choice of reagents.-An inhibitor for injection into nerves should be one which is capable of forming a persistent compound or adsorbate at the site of injection, and which is highly toxic at low concentrations. Fixatives and certain powerfully adsorbed dyestuffs satisfy these conditions, and the majority of the substances whose use is described below come under the former category. Apart from fixatives, the effects of certain other reagents have been examined ; and though their selection may be regarded as arbitrary, some sort of theoretical justification for their use could be brought forward in each case. Sodium taurocholate, for instance, is powerfully surface-active, and should therefore inhibit very strongly the amœboid movement which takes place at the tip of the growing axon (Speidel 1932, 1933). Again, aldehydes as a class are powerful inhibitors of growth and cell movement (Boyland and Mawson, 1938 ; Pomerat and Willmer, 1939; Medawar, 1940). Substances other than fixatives have however proved unsuccessful in practice, and in some cases have led to highly undesirable secondary reactions which are uniformly lacking when fixatives are used. This may be because fixatives are themselves "fixed" by the tissues they act on, so that their destructive effects are sharply local.

Fixatives are themselves a very miscellaneous collection of compounds, but in some cases it has proved possible to correlate their physico-chemical properties with their action in vivo.

\section{Summary of Results}

Long-period controls.-Huber and Lewis noted the formation of end bulbs or spindle-shaped enlargements in all but one of their set of 20 controls, the exception being the longest-period animal (1 year) in which complete reunion between the stumps had taken place. Large end bulbs (some of which are illustrated in Fig. 2, 1 to 6) have been found in all but one of the 12 long-period controls laid down by us, and in 6 , equally distributed throughout the age series, a resection gap of 30 to $40 \mathrm{~mm}$. (15 to $2 \$$ times the diameter of the intact nerve) had beet bridged by new growth and fibres had entered the peripheral stump (Fig. 2, 1, 4, 6). As a general rule, the largest bulbs, 4 to $6 \mathrm{~mm}$. in maximum width, were found on central stumps with no morphological connections to the peripheral stumps; and these showed the heaviest adhesions to muscle and fascia. The detailed anatomy of neuromas of section has been investigated by Dustin (1918), Cone (1918), Huber and Lewis (1920), and many others. Tracing the fibres from the centre towards the periphery we may usually distinguish (a) a normal zone of parallel fibre orientation ; (b) a " flare " zone, corresponding to the central end of the bulb, where the fibre tracts fan out symmetrically and there is marked proliferation of endoneurial connective tissue (c) a plexiform zone, where small bundles of fibres undergo chaotic deviations and criss-crossing, often turning back on themselves ; and finally $(d)$ a zone of involvement, where a comparatively small number of fibres tend to reassume a longitudinal orientation and invade fascia and muscle. Alternatively, $(d)$ may be represented by a thin strand of tissues uniting the central with the peripheral stump. A "typical" neuroma of section is illustrated by Fig. 3, and some of the essential features in its anatomy appear in examples of injection with ineffective reagents (see below).

For a proper understanding of the mechanism of inhibition, two points deserve emphasis. The first is that the greater part of the neuroma (unlike the glioma of the peripheral stump) forms within the nerve, i.e. within the compass of the original 

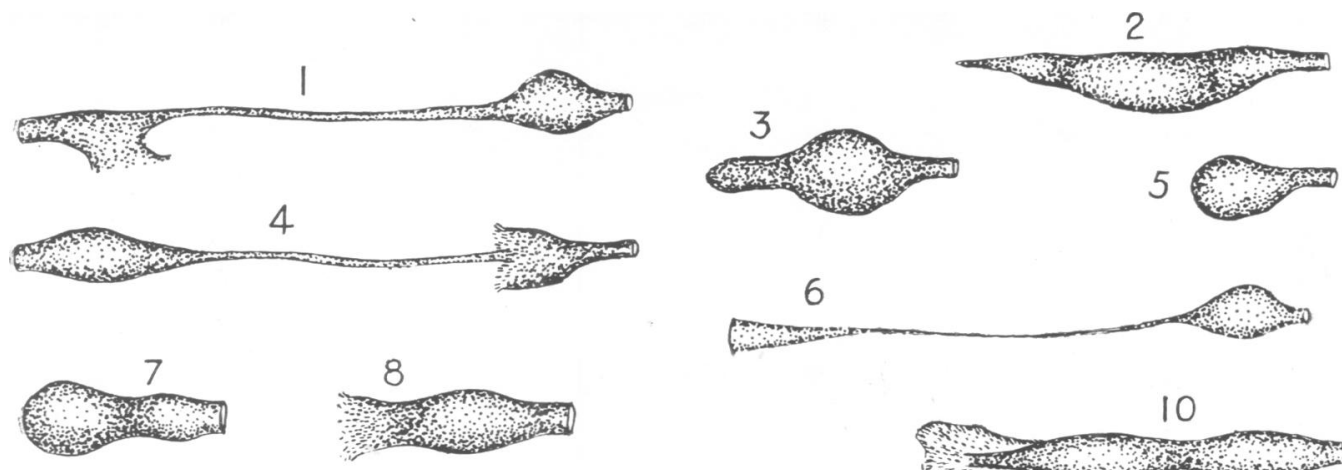

8
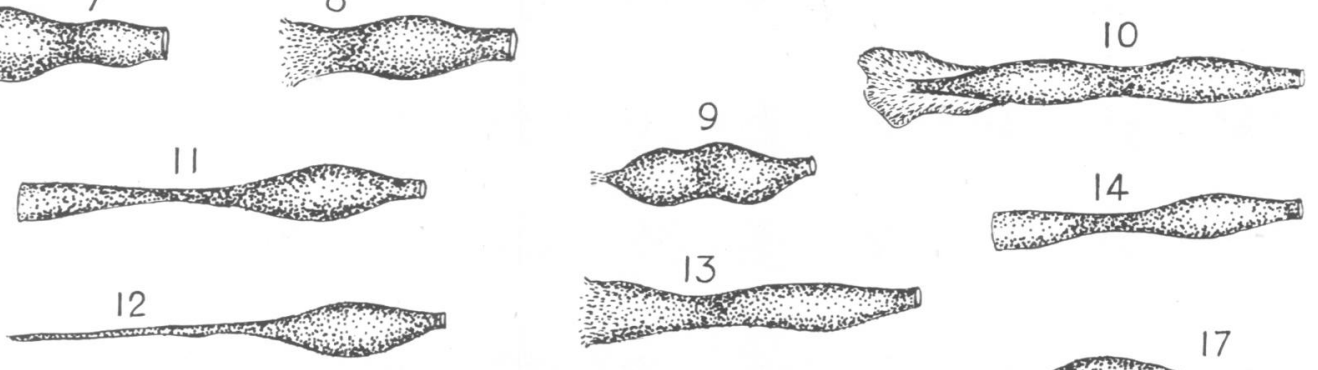

13

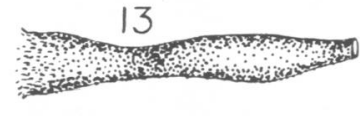

17

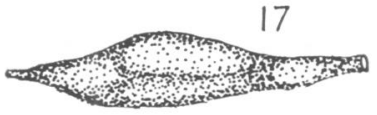

$15 \quad 16$
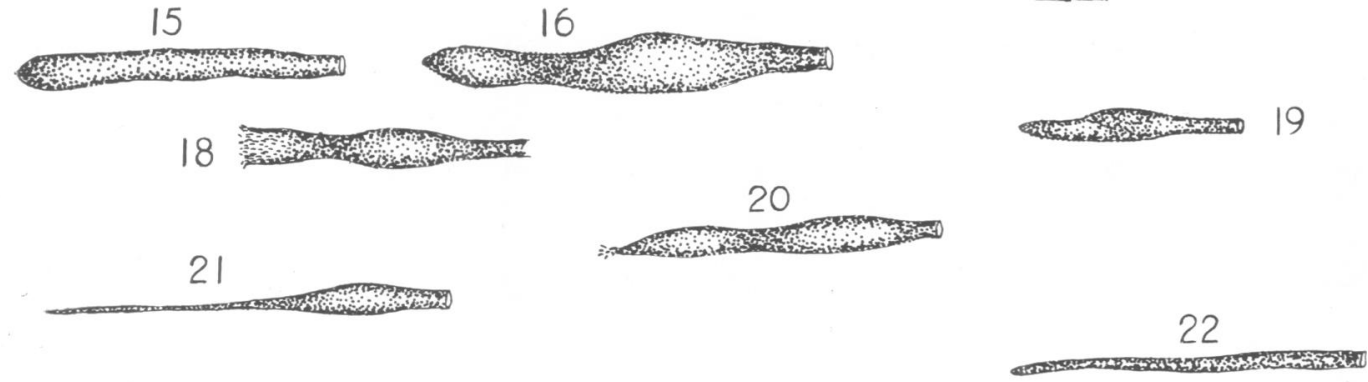

23

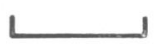

$10 \mathrm{~mm}$.

FIG. 2.-Varieties in the conformation of the central stump of the tibial nerve in the rabbit after simple section (1 to 6) and after injection (7 to 24$)$.

In $1,4,6,11$, and 14 the central and peripheral stumps have reunited: the central stump lies to the right.

1 to 6: Simple neuromas of section after (1) 200 days (reunion); (2) 389 days; (3) 457 days; (4) 117 days (reunion); (5) 103 days; (6) 165 days (reunion).

(7) 58 days after the injection of heptyl aldehyde; (8) second neuroma formed 179 days after the removal of 7; (9) 109 days after the injection of heptyl aldehyde (another specimen); (10) 330 days after the injection of crotonaldehyde: heavy muscle adhesions at the tip; (11) 61 days after the injection of 10 per cent. uranium nitrate: the stumps reunited; (12) 462 days after the injection of 10 per cent. formaldehyde; (13) 493 days after the injection of 2 per cent. osmic acid; (14) 62 days after the injection of 4 per cent. sodium taurocholate in absolute alcohol: a short resection gap bridged; (15) 60 days after the injection of a saturated solution of iodine in absolute alcohol: an even terminal swelling; (16) 95 days after the injection of a 4 per cent. solution of sodium taurocholate in absolute alcohol; (17) 169 days after the injection of 2 per cent. tannic acid in absolute alcohol; (18) 97 days after the injection of absolute alcohol; (19) 124 days after the injection of sodium phenate (10 per cent. phenol); (20) 207 days after the injection of a saturated aqueous solution of picric acid; (21) 121 days after the injection of a saturated aqueous solution of mercuric chloride: a small bulb has formed above the tapering stretch, which does not represent new outgrowth; (22) 259 days after the injection of 40 per cent. formaldehyde; (23) 237 days after the injection of 1 per cent. gentian violet; (24) 55 days after the injection of 2 per cent. tannic acid in 40 per cent. formaldehyde: the last $15 \mathrm{~mm}$. consists of fixed and dead nerve uninvaded by new fibres. 


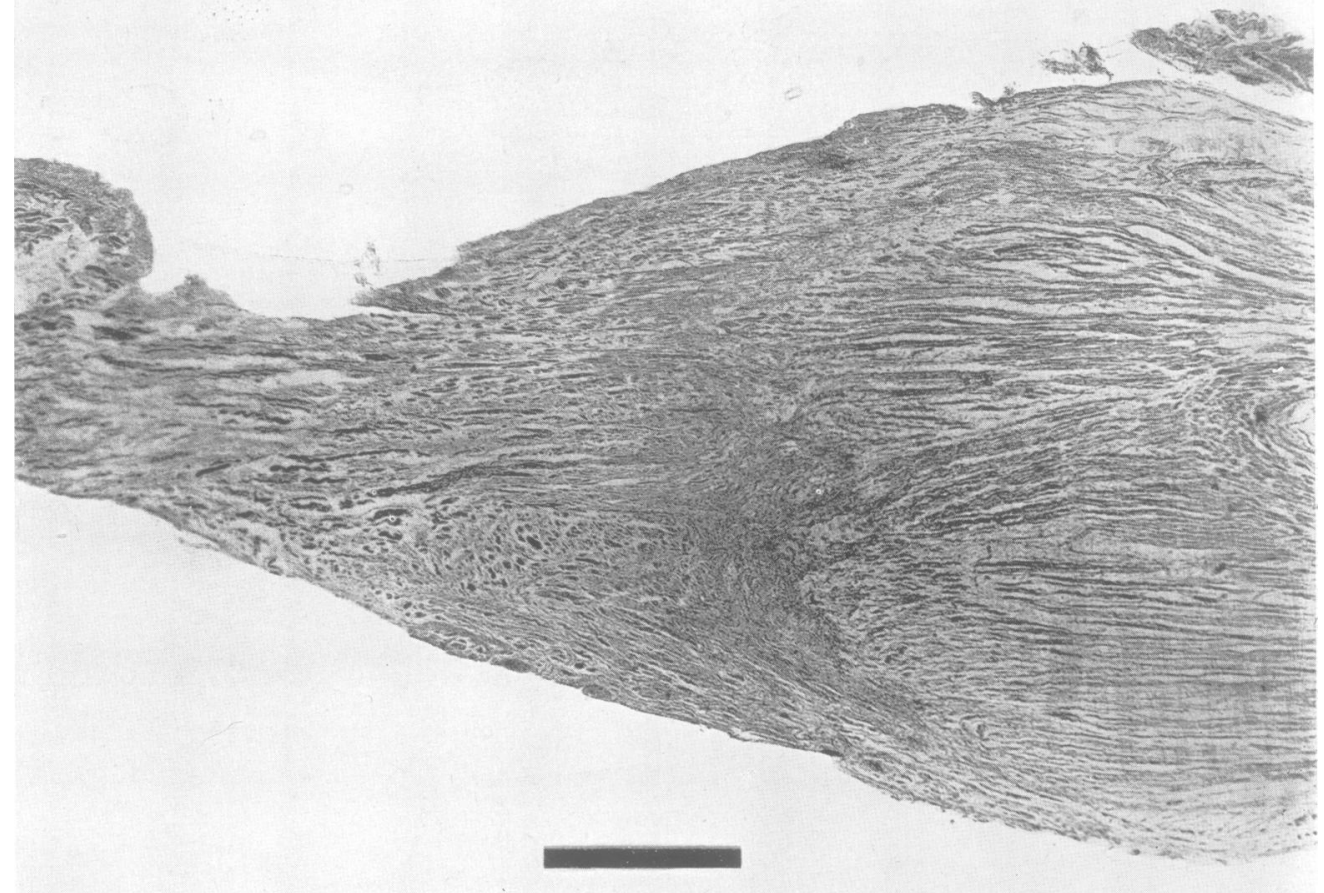

FIG. 3.-The distal half of a 457-day neuroma of section: scale $1 \mathrm{~mm}$., Holmes' stain. The central end lies to the right. Note the abrupt transition between the "flare" and plexiform zones. Beyond the plexiform zone, aco comparatively small number of fibres have readopted a parallel orientation and invaded fascia and muscle.

epineurial sleeve, and not by the proliferation of cells which have grown out from the end. This is one of the reasons why the mere closure of the central stump, whether by chemical or surgical means, is inadequate. The second is that as a general rule the end of the neuroma is anatomically speaking open, i.e. the epineurium does not form a complete investment for it. Encapsulated neuromas in the rabbit are rare. The state of the central stump, whether closed or open, is one of the grounds used below for distinguishing between successful and unsuccessful inhibitions.

Chemical inhibitions: Full records are available for 87 experiments, which are divided up as follows :

Alcohol, 70 to 100 per cent.: 12 experiments, 45 to 189 days, including experiments in which alcohol was used as a solvent for sodium taurocholate and tannic acid (Fig. 2, 14, 16, 17, 18).

Alcohol has been a failure in our experiments. In 5 out of the 12 experiments (cf. Fig. 2, 14) short resection gaps had been bridged by nerve fibres. All twelve show central swellings of various sizes-smaller where reunion had taken place between the stumps. Heavy adhesions between the central stump and underlying fascia and muscle were the rule. Traces of fixed and unremoved debris have been found in the nerve after three months. In the formation of a bulb, the open state of the central stump, the existence of a plexiform zone and a zone of involvement, the long-period alcohol neuroma closely resembles and cannot be distinguished with certainty from a normal neuroma of section.

It seems justifiable to attribute the greater success of alcohol injection in Huber and Lewis's 28 experiments (11 to 157 days) to the fact that they used multiple injections, often widely separated on the nerve, and that a much greater length of nerve was therefore destroyed. Although the technique of multiple injection might very well be used in clinical practice, our experiments show quite clearly that under uniform conditions, using singlepoint injections of not more than $0.2 \mathrm{ml}$. of each reagent, alcohol is notably less successful than the other coagulants we have used. It is noteworthy that in the last four (62 to 137 days) of Huber's 12 additional experiments in which the nerve was left uncut after injection with alcohol, fibres were found to have passed through the coagulated stretch and entered the peripheral stretch of the nerve.

Tannic acid, 2 to 4 per cent.: Aqueous and alcoholic solutions, 11 experiments, 29 to 189 days (Fig. 2, 17, Fig. 8, 1, 2).

Short resection gaps were bridged in 3 experiments, and in these neuromas, though present, were comparatively small. Large neuromas (three or more times the diameter of the normal nerve) were found in 6 cases; and in these 9 taken together, the stumps were heavily involved in fascia and muscle. Two short-period animals $(31,33$ days) showed a temporarily successful inhibition, and histological examination showed that the end of the nerve was sealed off with a fixed plug (Fig. 8, 1, 2) into which new fibres had not yet penetrated. In other experiments there was no evidence that the nerve had been completely impregnated with tannic acid. This fact is referred to again below.

Formaldehyde, 10 to 40 per cent.: 21 experiments 
FIG. 4-About 8 months after the subepineurial injection of 40 per cent. formaldehyde: scale $1 \mathrm{~mm}$, Bodian's stain. The ground plan is similar to that illustrated in Fig. 5, but should be contrasted with that illustrated in 0 Fig. 6. Note that the stump is closed by its epineurial sheath, which is lightly adherent to fascia. No fibres escape. $\vec{\circ}$
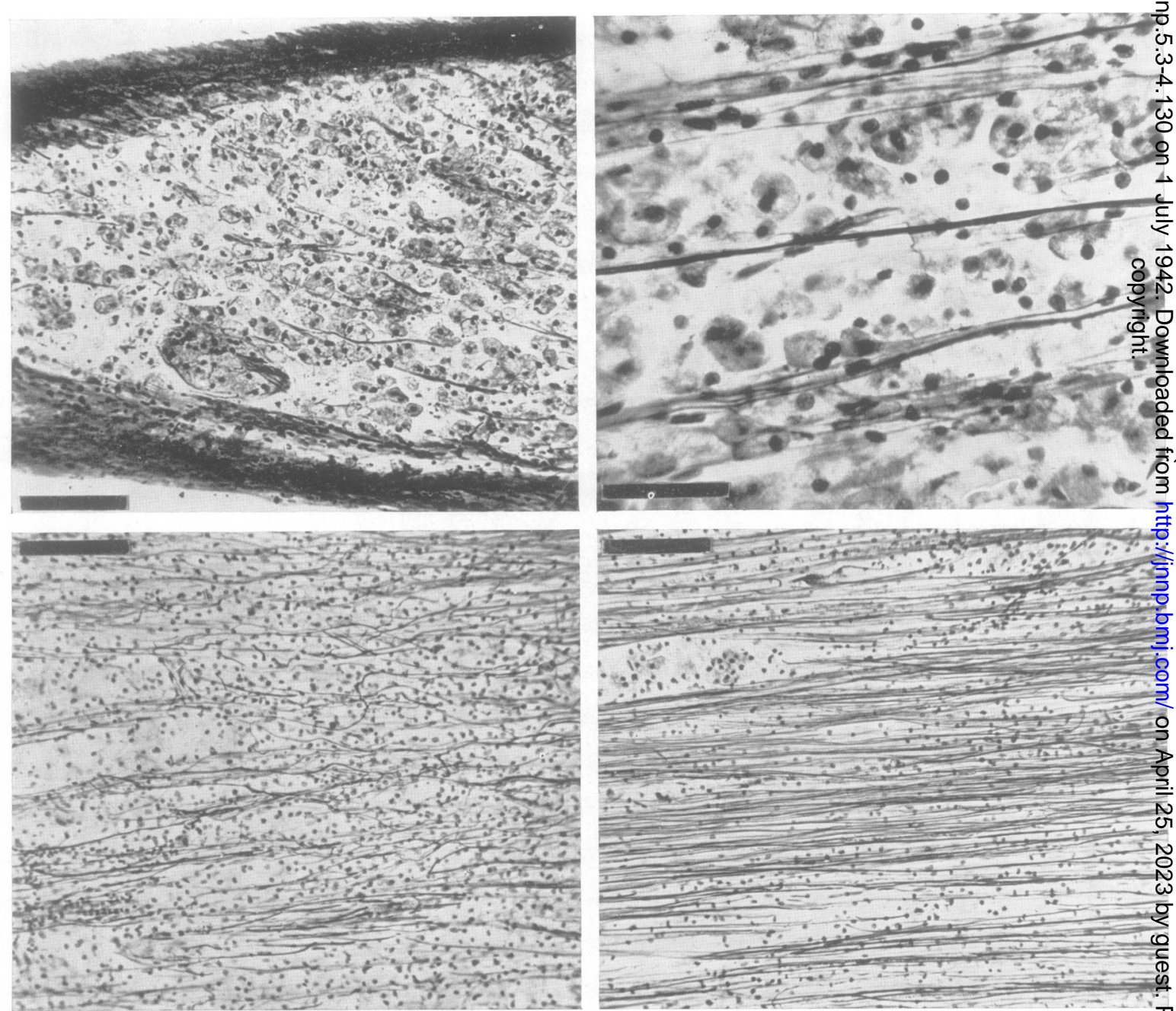

FIG. 5.-Illustrating the general anatomy of the central stump about 3 months after injection with 40 per cent. $\frac{\bar{O}}{\vec{P}}$ formaldehyde: Bodian's stain. (1) Scale $0.1 \mathrm{~mm}$. Just behind the tip. A few fibres have penetrated into a dense collection of giant macrophages. (2) Scale $0.05 \mathrm{~mm}$. In the zone of (1), under higher power. (3) Scale $0 \cdot 1 \mathrm{~mm}$. $\overrightarrow{\mathbb{D}}$ About $10 \mathrm{~mm}$. behind the tip: zone of fibre deviation. (4) Scale $0 \cdot 1 \mathrm{~mm}$. About $20 \mathrm{~mm}$. behind the tip: macrophages $\varrho$ still abundant. 
29 to 462 days. (Fig. 2, 12, 22, 24; Fig. 4, 5, 6.) These are divided up as follows:

10 per cent.: 5 experiments, 68 to 462 days. Resection gaps bridged in two cases. All 5 show bulbous enlargements of the central stump with moderate adhesions.

20 per cent.: 3 experiments, 72, 297, 356 days; 40 per cent.: 9 experiments, 29 to 335 days; 40 per cent. in 5 per cent. tannic acid: 4 experiments, 29 to 84 days. Experiments of this second group may be considered together. Single point injections of 20 to 40 per cent. by an extremely sparse stroma of connective tissue. $C$ Fig. 5, 1 to 4, illustrates three zones in a relatively short period (3 months) injection with 40 per cent. formaldehyde: a central zone of normal fibres orientated parallel to one another (4); a branching zone (3); and a zone of decreasing fibre density $(1,2)$ where the majority of phagocytes are accumulated. The condition is not substantially different after 8 months (Fig. 4), though macrophages are somewhat fewer. There is no swelling of the nerve, and no evidence of adhesion except at the
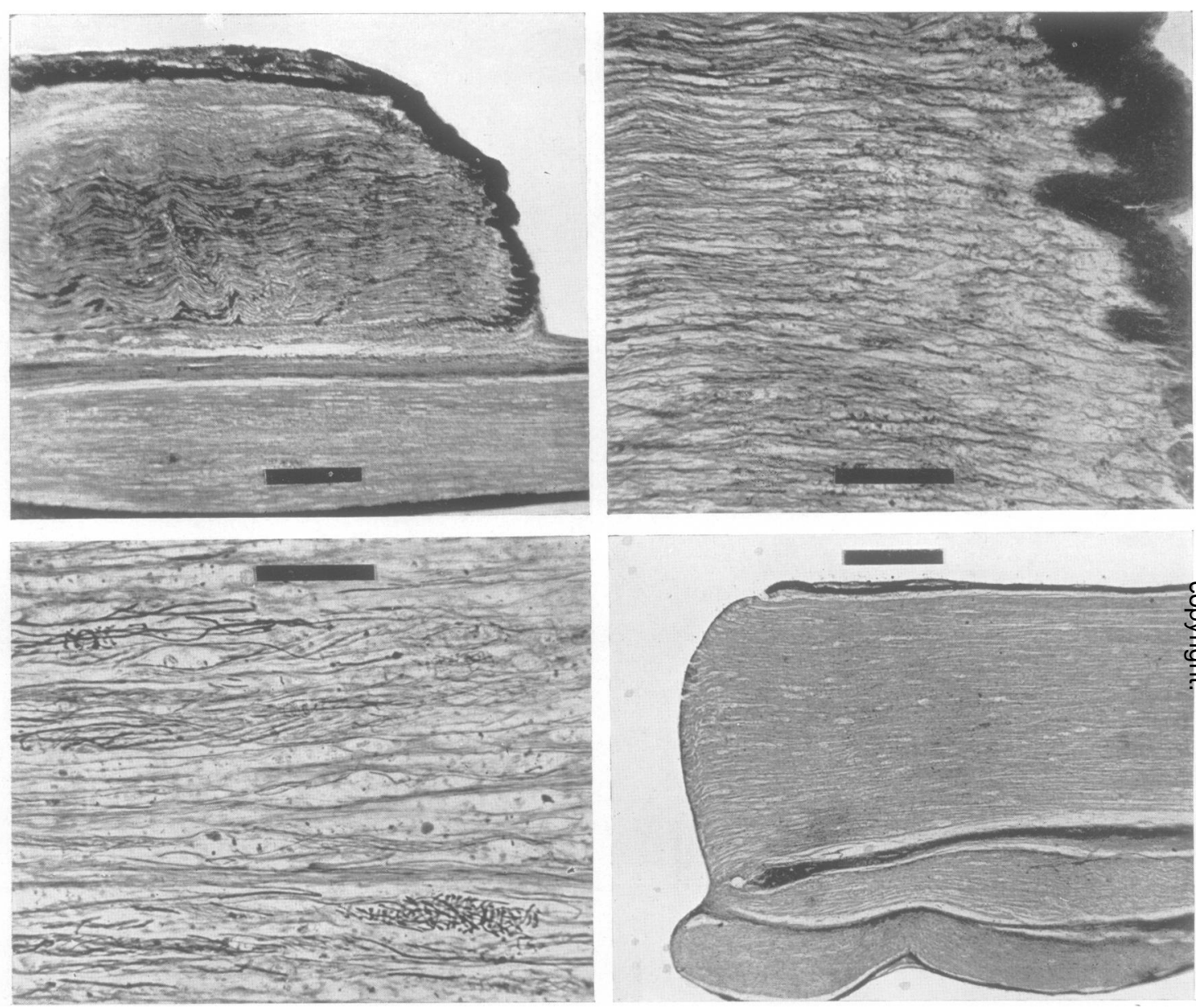

Fig. 6.-Illustrating the general anatomy of the central stump 2 to 3 months after the intraneural injection of 40 per cent. formaldehyde. Contrast with Fig. 5. Bodian's stain. (1) Approximately 2 months after injection: scale $0.5 \mathrm{~mm}$. The last $15 \mathrm{~mm}$. of the nerve is fixed and dead, though the epineurial sheath has closed over the tip. The peroneal nerve, running alongside, has been damaged but not destroyed by the injection. (2) Scale $0 \cdot 1 \mathrm{~mm} .:$ the tip, more highly magnified. Fixed and empty remains of the original endoneurial scaffolding. (3) About $15 \mathrm{~mm}$. behind the tip: scale $0.1 \mathrm{~mm}$. The most advanced zone of new fibres in the dead tissue: deviation and spiralling. (4) About 3 months after intraneural injection: another specimen, scale $0.5 \mathrm{~mm}$. Note the abrupt termination of the nerve.

formaldehyde have proved strikingly successful. No bulbs are formed. The nerve, which is yellow and lacks its usual sheen, tapers down from its normal width for a length of $30 \mathrm{~mm}$. to a tip lightly adherent to underlying fascia (Fig. 4). Elsewhere the nerve is wholly non-adherent and may be picked up freely from its fascial bed. There is no fibrosis, either in the nerve or in surrounding tissues. Histological examination shows a clear-cut distinction between subepineurial and intraneural injection. In the former case, to which the remarks made above apply, the yellow tapering stretch is found to contain nerve fibres which thread their way through a mass of giant mononuclear cells supported extreme tip. The stump is closed by its own epineurial sheath.

After intraneural injection, the histological picture (Fig. 6, 1 to 4) is somewhat different. The nerve ends off abruptly with a fixed plug $(1,2,4)$ towards the central end of which (3) macrophages are in action and fibres are beginning to invade the dead tissue. Unfortunately, we have no long period experiments with intraneural injection.

Gentian violet, ${ }^{*} 1$ per cent. aqueous: 2 experiments,

* The name given to an ill-defined mixture of the more highly methylated violet pararosaniline dyes (methyl violet, crystal violet, etc.). It may be assumed that triple-dye (gentian viloet, acriflavine, brilliant green mixture) would give results comparable to those obtained with gentian violet.

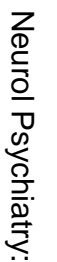


217 and 237 days. Subepineurial injection (Fig. 2, 23; Fig. 7).

The nerves tapered down from their normal width over distances of 30 and $60 \mathrm{~mm}$. respectively, and were nowhere bulbous or swollen. The last $5 \mathrm{~mm}$. were discoloured with unremoved gentian violet, and there were in each case light attachments at the tip. Longitudinal sections of the longer-period specimen showed extensive accumulations of giant phagocytes at the tip (Fig. 7), the last $10 \mathrm{~mm}$. being collagenized and feebly penetrated by isolated new nerve fibres. It is not possible to say with certainty whether the greater part of the collagen at the tip represents fixed remains from the original injection, or whether it is collagen of new formation.

Higher up, the nerve fibres are clearly threading their way through the remains of fixed endoneurial scaffolding. The material has the appearance of normal regenerating a plug of fixed tissue into which fibres had not penetrated and in which phagocytosis by giant cells had hardly begun. These facts are discussed below.

Non-fixatives: Twenty-two experiments ranging from 29 to 539 days are divided among quinine hydrochloride ( 1 per cent.), optochin ( 1 per cent.), antipyrene ( 5 per cent.), sodium taurocholate (4 to 10 per cent. in absolute alcohol), beryllium nitrate (10 per cent.), crotonaldehyde and heptylaldehyde (10 per cent. in absolute alcohol). (Fig. 2, 7, 8, 9, 10, 14, 16).

As a class, non-fixatives have given uniformly unsuccessful results, and they throw very little light on the mechanism of inhibition. The first three may be regarded as having no significant effect from the point of view of fibre inhibition, while Salm (1940) has shown that quinine elicits a prompt fibroblast reaction shortly after injection. Crotonaldehyde and heptylaldehyde are in no way comparable to formaldehyde; they are un-

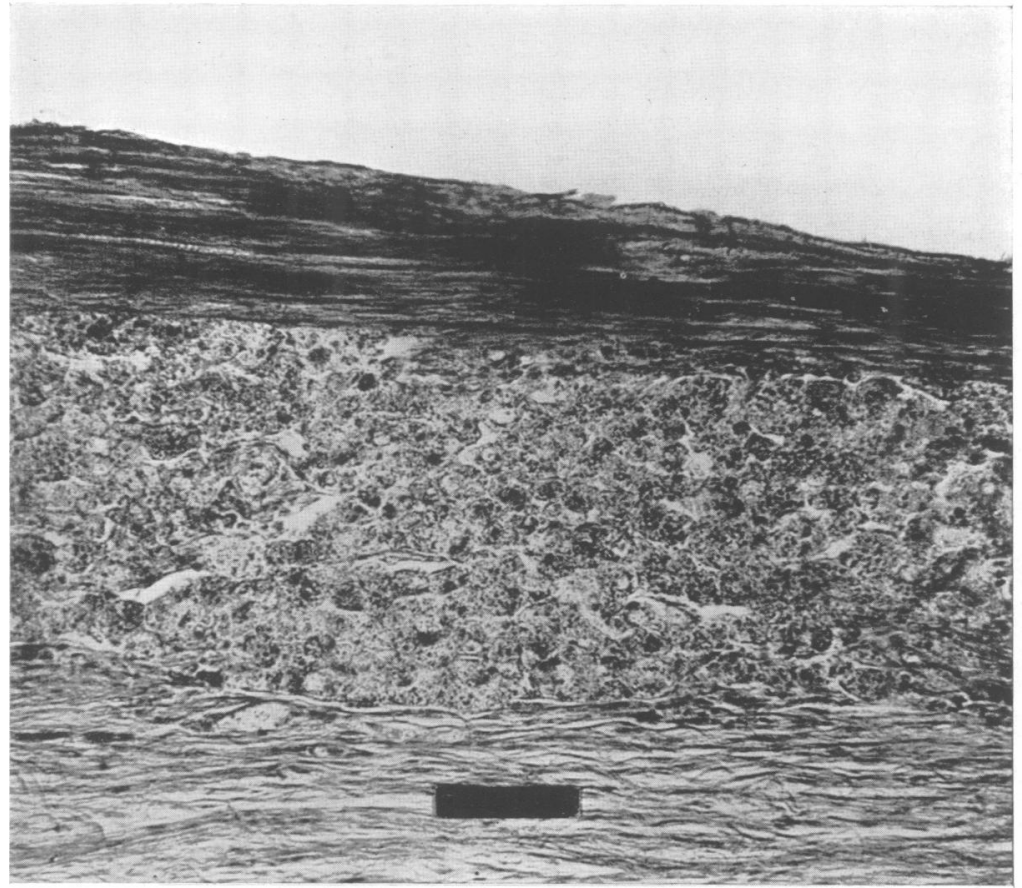

Fig. 7. $-15 \mathrm{~mm}$. behind the tip of the nerve 8 months after injection with 1 per cent. gentian violet. Scale $0.075 \mathrm{~mm}$., Bodian's stain. A densely packed collection of macrophages occupies about one-third of the diameter of the nerve in sectional view.

nerve only about $30 \mathrm{~mm}$. from the tip, and macrophage islands are present at least $60 \mathrm{~mm}$. from the tip.

Other coagulants: Chromic acid (1 per cent.), mercuric chloride (sat. aq. soln.), sodium phenate (in the form of the proprietary preparation "phenoda," containing 10 per cent. phenol as phenate), picric acid (sat. aq. soln.), iodine ( 5 per cent. absolute alcohol), and uranium nitrate (10 per cent. aqueous) together account for 20 experiments ranging in length from 23 to over 500 days. (Fig. 2, 13, 15, 19, 20, 21; Fig. 8, 3, 4).

All give results inferior to formaldehyde and gentian violet, though the data for chromic acid ( 2 experiments: 36 and 119 days) are incomplete. Iodine and uranium nitrate inhibit only temporarily, but picric acid, sodium phenate, osmium tetroxide and in particular mercuric chloride may be said to give marked and significant inhibition for periods up to 150 days.

The single medium period (119 days) chromic acid injected nerve was found to be soft and "puffy" in appearance, and of normal width down to an abrupt pencil-point termination. On microscopical examination (Fig. 8, 3) the terminal stretch was found to consist of pleasant to handle and give rise to moderate fibrosis and other secondary reactions. Beryllium nitrate is likewise ineffective, while giving rise to a most violent and undesirable fibroblast proliferation.

\section{Evaluation of Results}

In judging between the efficacy of the various reagents listed above, the question of neuroma formation is clearly not the only one to be considered. Nor, since the experiments range over a considerable period, is it possible merely to record the presence or absence of fibres in the injected stretch. We will leave aside the total failure represented (as in 5 out of 12 experiments with alcohol) by functional reunion of the stumps, since this does not by any means occur invariably in the controls. In the main, the inhibitions described as "successful ", (a term which applies to formaldehyde and 

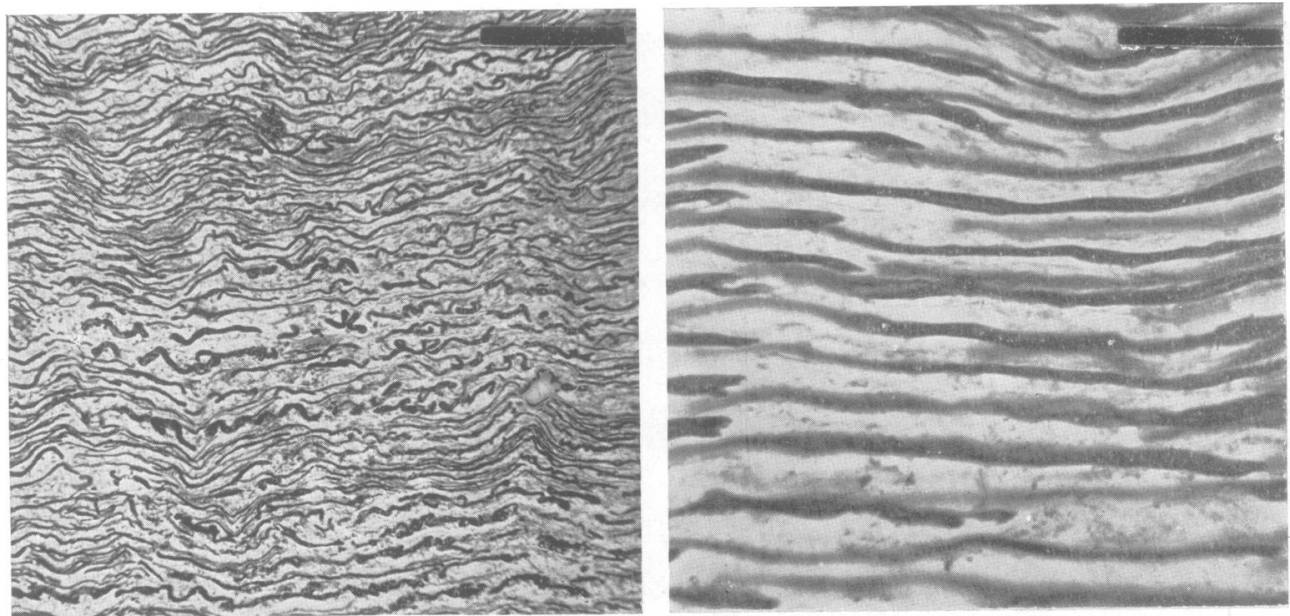

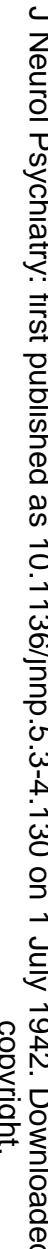

FIG. 8.-The effect of fixation with tannic and chromic acids. Bodian's stain. (1) One month after injection with 4 per cent. tannic acid, just behind the tip of the nerve: scale $0.1 \mathrm{~mm}$. (2) The same: scale $0.05 \mathrm{~mm}$. The axons are fixed and dead: no living element is present. (3) and (4): 4 months after the injection of 1 per cent. chromic acid: scale $0.1 \mathrm{~mm}$. In (3), macrophages are removing the fixed cellular debris just behind the tip of the nerve, but no axons have yet arrived. In (4), $5 \mathrm{~mm}$. behind the tip, regenerating axons are beginning to invade the terminal plug.

gentian violet experiments, and with greater reserve to medium-period experiments with some of the other fixatives) are distinguished from the controls and the simple failures-reagents which failed without giving rise to secondary reactions-in the following combination of ways :

\section{State of the Central Stump}

\section{Successful inhibitions}

1. Axons decrease in number towards the periphery.

(This is clearly shown by Figs. 4, 5, and 6. The fact that some fibres reach the tip is of secondary importance. They gradually fall off in number until only one or two are left.)

2. Absence of all but trivial and superficial adhesions; closure of the central stump; no escape of fibres.

\section{Failures and controls}

Axons increase in number towards the periphery, as a result of branching and convolution.

Heavy and intimate adhesions to fascia and muscle; central stump typically open; invasion of neighbouring tissues by nerve fibres.

\section{Successful inhibitions}

3. No neuroma: the nerve is equal to or less than its normal width throughout its length.

4. No gross fibre-bundle deviations, "flaring," convolution and other signs of unrestricted free growth.

5. Endoneurial connective tissue reaction lacking.

If these criteria are applied to the experimental results, as they have been above, they clearly distinguish formaldehyde and gentian violet from the other fixatives; and fixatives as a class from nonfixatives.

\section{The Theory of Inhibition by Coagulants}

The case of alcohol is simplest and will be considered first. Coagulation by alcohol, like that brought about by heat, is thought to consist essentially of dehydration of the colloidal particles without secondary reactions. Alcohol fixed proteins are in

Failures and controls Neuroma formation in-

Flaring, whorling and convolution of fibre bunplexus

Proliferation of endoneurial connective tissue. 
no way resistant to proteolytic digestion; moreover alcohol is not only a most feeble penetrant of tissue (Underhill, 1932; Medawar, 1941a) owing to its high fixing concentration, but any excess of it that remains will be rapidly metabolized away. It follows that alcohol destroys nerve without offering much more than a temporary mechanical obstacle to regenerating fibres. There is no theoretical reason why it should be a particularly effective inhibitor, and in fact it is not:

Tannic acid, chromic acid, and gentian violet share in common the property of making the proteins they fix totally resistant to tryptic digestion (Medawar, 1942), and the first two at least, for which further data are now available, likewise stabilize proteins to digestion with papain, which, like the tissue cathepsins, acts in a faintly acid environment. They therefore offer a chemical as well as a mechanical obstacle to axon regeneration.* Examples of tannic acid and chromic acid fixation (Fig. 8) .show that highly resistant chemical seals can be formed by their action. Unfortunately, tannic acid penetrates tissue so feebly (see Medawar, 1942) that it is most unlikely that the whole thickness of the larger human nerves could be coagulated by it. Even the failure of tannic acid fixation of the tibial nerve of the rabbit seems to be due in part to incomplete fixation. But it is not by any means certain that the formation of a mechanically perfect seal at the end of the nerve is a desirable reaction. Experiments on ligation (for this treatment amounts to chemical ligation) and encapsulation of the central stump with collodion sheaths show that neuromas are not prevented from forming, whether fibres can just squeeze their way out or not.

The evolution of formaldehyde-fixed central stumps appears to fall into the following stages : a latent period, the presence of which we can take for granted ; the slow mobilization of giant mononuclear phagocytes to break down the coagulated tissue ; the invasion of the fixed tissue with axons, probably at first outstripping their Schwann cells; and finally - a stage which has not been reached in practice-the complete colonization of the stump with myelinated fibres. The invasion process, which is well under way before 100 days (Fig. 5) is by no means complete at 300 (Fig. 4); and the inference is that the inhibited stretch is permeable to fibres but highly unfavourable to their growth. Why formaldehyde should be so successful is not clear. It is not a precipitant, and fixation by it does not interfere with subsequent proteolytic digestion. It is however a most powerfully reactive substance, which condenses with a great variety of compounds other than proteins, and it is quite possible that its action on nerves is due to properties other than those of mere protein fixation.

The action of gentian violet is easier to understand. In the absence of a circulation at the end of the central stump, the conditions there may be likened

* It should be noted that the polymorphs and lymphocytes of the rabbit lack tryptic-type enzymes (Barnes, 1940) and the giant cells referred to in the text may do so as well. to a supravital tissue culture. Gentian violet is strongly "adsorbed" on to living tissues, and under in vitro conditions is an intensely powerful protoplasmic poison.* It is liberated in its original form extremely slowly (e.g. gentian violet precipitated proteins will still colour standing water after more than 2,000 hours continuous washing), and the proteins themselves are totally resistant to digestion by tryptic-type enzymes (Medawar, 1942). Since traces of gentian violet have been found in injected stumps after more than 200 days, we may assume that it is still acting as an inhibitor at that period. Gentian violet has the advantage of being highly coloured; and in clinical practice its antiseptic action could be extended to gram negative organisms by combining it with acriflavine and brilliant green in the form of the mixture known as triple-dye.

Coagulation by the other reagents listed above, and more particularly by the salts of heavy metals; need not be considered in detail, though it throws a clear light on the mechanism of inhibition. The proteins they fix are not resistant to proteolysis, but the highly toxic heavy metal ion is probably liberated during digestion. Whatever success they have in practice cannot therefore be attributed merely to killing the central stump of the nerve and plugging it up. It must be due, to a far greater extent, to the steady maintenance of an environment which is permeable, but only just permeable, to regenerating fibres. The reaction is one of chemical defence in depth.

Fibre-inhibition, neuroma formation, and adhesions

The ligation experiments described earlier in the paper, and the qualitative observation that neuromas of section tend to be smaller where a thick functional bridge is established between the stumps, suggest that neuroma formation, although it might be an independent traumatic reaction on the part of Schwann and connective tissue elements in the nerve, does in fact depend upon the presence of downgrowing axons. This conclusion is exactly confirmed by the experiments on chemical inhibition reported above. When fibres are absent, or merely penetrate in small numbers to the tip, no neuroma is formed. For this reason the presence or absence of bulbs may be used as an index of the success of inhibition.

A much less obvious consequence of fibre inhibition is that the heavy and intimate adhesions between the central stump of the nerve and neighbouring tissues which are invariably found in control experiments and after ineffective inhibitions are wholly lacking in the "successful" inhibitions. Here again, there is no very obvious reason why adhesions should not be set up by the connective tissue elements in the nerve; but this proves not to be the case. Where fibres are absent or present in trivially small numbers in the inhibited stretch, adhesions are likewise lacking, or are confined to

* Tests by the methods of Jacoby, Medawar and Willmer (1941), section II, and Medawar (1941b) give gentian violet a " threshold" toxicity to fibroblasts below $1: 100,000$. 
a light epineurial attachment at the tip of the nerve. Elsewhere, the nerve disengages freely from its fascial bed. There has been no exception to the rule that where no neuroma is formed, adhesions are superficial or absent.

\section{Clinical Application}

Injection with coagulants destroys a relatively greater length of nerve in laboratory animals than it does in men. The absolute length is however more important, if the reasoning of a previous section is correct ; so that the experimental findings can be translated into clinical terms without undue reserve. It is most unlikely that human nerves behave much differently from those of the rabbit with regard to such an unspecific procedure as chemical coagulation.

Experience in injecting the nerves of the rabbit suggests that for the best results the following details should be observed :

(a) The nerve should be carefully packed with moist absorbent gauze before injection.

(b) Injection should be both intraneural and subepineurial.

(c) Steps should be taken to control the distance the injection fluid travels up the nerve. The injection fluid should be coloured; with methylene blue in the case of formaldehyde. A light ligature some centimetres below the lowest working branch of the nerve, later removed, should prevent the injection fluid travelling further up than is intended.

(d) Since there must always be the danger of a leak from the nerve after injection, it should be replaced where it can do little harm, and in no case in contact with another nerve.

Indications and contraindications for the use of injection methods naturally fall outside the scope of this paper. But whatever reasoning justifies the removal of a neuroma for the attempted relief of pain likewise justifies the prophylactic use of $z$ injection in order to prevent it forming again. We conclude from these experiments that formaldehyde (20 to 40 per cent.) and gentian violet (1 per cent.) are suitable reagents for this purpose, while alcohol, which has hitherto been so widely used, is relatively ineffective. It should not be forgotten, however, that both formaldehyde and gentian violet are intensely poisonous substances; and we do not suggest that they should replace alcohol for delicate injections, such as those of the Gasserian ganglion, where even the use of alcohol is attended with sufficient danger.

\section{Summary}

1. Chemical and surgical methods for preventing the regenerative growth of nerve fibres from the central stump of a divided nerve are discussed and reviewed.

2. An attempt has been made to evaluate chemical methods of inhibition by a uniform series of injections of fixatives and non-fixatives into the tibial nerve of the rabbit.

3. Fixatives as a class are superior to nonfixatives. Formaldehyde, in concentrations of 20 per cent. upwards, and 1 per cent. gentian violet prevent the formation of end-bulbs and the develop- $c$ ment of adhesions between the nerve and its bed for $\rightleftharpoons$ at least a year. Alcohol is only effective for periods $\vec{\omega}$ below two months. Other fixatives occupy an intee $\mathrm{N}$ mediate position.

4. Neuroma formation and the development 8 strong adhesions between the stump of the nerve and its bed do not occur in the absence of regenerating nerve fibres.

One of us (L.G.) is indebted to the Nuffield Committee for the Advancement of Medicine for a personal grant. We are grateful to Prof. H. J. Seddon and Mr. J. Z. Young for their criticism and advice.

\section{REFERENCES}

Bardenhäuer, E. (1908). Dtsch. Z. Chir., 96, 128. Barnes, J. M. (1940). Brit. J. exp. Path., 21, 264.

Benedict, W. (1922). Klin. Z. Streitfragen, 6, 3. Bersou, W. (1912). Névraxe, 13, 581.

Beswerschenko, A. P. (1929). Z Zbl. Chir., 56, 455.

Bodian, D. (1936). Anat. Rec., 65, 89.

Boyland, E., and Mawson, E. H. (1938). Biochem. J., 32, 1982.

Chapple, W. A. (1918). Brit. med. J., 1, 399.

Cone, S. M. (1918). Brit. J. Surg., 5, 524.

Corner, E. M. (1918a). Ibid., 6, 273.

(1918b). Proc. roy. Soc. Med., R.S.M. lectures, 11, 7.

Dustin, A. P. (1918). Ambulance de l'Ocean, 1, 71.

Erlacher, P. (1924). Arch. orth. Chir., 23, 287.

Finkelnburg, H. (1903). Verh. Kongr. inn. Med., 24, 75. (1907). Dtsch. med. Woch., 40, 1665.

Foerster, O. (1929). Therapie der Schussverletzungen der Periph. Nerven, Berlin, 1703-1708.

Fraenkel, E. (1884). Berl. klin: Wschr., 15, 184.

Frankenthal, L. (1928). Btr. klin. Chir., 143, 237.

Gutmann, E., Guttmann, L., Medawar, P. B., and Young, J. Z. (1942). J. exp. Biol., 19, 14.
Harris, W. (1909). Lancet, 1, 1310.

Hedri, A. (1921). Verh. dtsch. ges. Chir., 1, 50.

Holmes, W. (1942). J. Path. Bact., 54, 132.

Holmes, W., and Young, J. Z. (1942). J. Anat., 77, 63. 은

Huber, G. C. (1927). Med. Dept. U.S. Army, 11 (Surgery), 1117.

Huber, G. C., and Lewis, D. (1920). Arch. Surg., 1, 85. Jacoby, F., Medawar, P. B., and Willmer, E. N. (1941). N Brit. med. J., 2, 149.

Krüger. H. (1916). Munch. med. Wschr., 1, 368.

Laewen, A. (1925). Beitr. klin. Chir., 133, 405.

- (1930). Munch. med. Wschr., 2, 1667.

Lexer, E. (1931). Zbl. Chir., 1, 341.

May, O. (1912). Brit. med. J., 2, 465.

Marinesco, G. (1918). Proc. roy. Soc. Med., R.S.M. Lectures, 11, 5.

(1920). Philos. Trans. B, 209, 229.

Medawar, P. B. (1940). Proc. roy. Soc. London, B, T $129,332$.

(1941a). J. R. micr. Soc., 61, 46.

(1941b). Lancet, 2, 182.

- (1942). Ibid., 1, 350.

Moscowicz, A. (1918). Zbl. Chir., 32, 547. 
Neuber (1883). Quoted by Frankenthal (1928).

Patrick, H. T. (1912). J. Amer. med. Ass., 58, 155.

Pomerat, C. M., and Willmer, E. N. (1939). J. exp. Biol., 16, 232.

Salm, R. (1940). J. Pharmacol., 70, 245.

Sanders, F. K., and Young, J. Z. (1942). J. Anat., $76,143$.

Schlosser, H. (1903). Klin. Mbl. Augenheilk, 41, 244.
Seddon, H. J. (1941). Personal Communication. Sicard, J. A. (1916). Presse Méd., 24, 241.

Speidel, C. C. (1932). J. exp. Zool., 61, 279. (1933). Amer. J. Anat., 52, 1.

Stookey, B. (1922). Surgical and Mechanical Treatmen of Peripheral Nerves, New York.

Underhill, B. (1932). J. roy. Micr. Soc., 52, 113. Young, J.Z., and Medawar, P. B. (1940). Lancet, 2, 126. 